Classification

Physics Abstracts

$73.40 \mathrm{~L}-73.60$

\title{
Electroréflexion et ellipsométrie spectroscopique d'hétérostructures InGaAsP/InP et GaAlAs/GaAs
}

\author{
C. Alibert, Fan Jia Hua \\ Equipe de Micro-optoélectronique de Montpellier (E.M. $\left.{ }^{2}\right){ }^{*}$ ), \\ Université des Sciences et Techniques du Languedoc, place E. Bataillon, 34060 Montpellier Cedex, France
}

M. Erman, P. Frijlink, P. Jarry et J. B. Theeten

Laboratoire d'Electronique et de Physique Appliquée (L.E.P.),

3, avenue Descartes, 94450 Limeil-Brevannes, France

(Reçu le 25 juillet 1983, accepté le ler août 1983)

\begin{abstract}
Résumé. - On utilise deux techniques optiques sensibles non destructives et complémentaires : l'électroréflexion et l'ellipsométrie spectroscopique pour analyser des structures semiconductrices multicouches. On montre, sur un alliage quaternaire de GaInAsP, qu'il existe une bi-couche d'InP. Sur des empilements d'hétérostructures contenant des couches ultra-minces, on détermine la composition chimique des couches, les épaisseurs, la qualité des interfaces, les contraintes mécaniques et les principales transitions optiques. On mesure par électroréflexion les niveaux électroniques d'un double puits quantique de GaAs dans une matrice de GaAlAs.
\end{abstract}

\begin{abstract}
Two complementary, non destructive and sensitive optical technics (electroreflectance and spectroscopic ellipsometry) are used to analysed semiconductor multilayers. Ultra thin layer of InP is detected on InGaAsP quaternary alloy. On stacked heterostructures with ultra thin layers, chemical compositions, thicknesses, interfaces quality, mechanical stresses and the mains optical transitions are determined. Electronic levels of a GaAs double quantum well in a GaAlAs matrix are measured by electroreflectance.
\end{abstract}

\section{Introduction.}

De nombreux composants électroniques et optoélectroniques à base de semiconducteurs III-IV sont basés sur des hétérostructures formées d'empilements de couches dites minces (épaisseur de $100 \AA$ à $10000 \AA$ ) ou ultra minces (épaisseur inférieure à $100 \AA$ ). Ce type de structure en couche renforce l'importance des propriétés liées aux interfaces et à la surface par rapport aux propriétés volumiques des matériaux constituant chaque couche.

Une bonne compréhension des phénomènes physiques intervenant dans le fonctionnement de ces dispositifs nécessite la mesure des paramètres essentiels caractérisant ces structures multicouches : il s'agit de paramètres intrinsèques tels que épaisseur, nature chimique, dopage de chaque couche, et de paramètres extrinsèques tels que états de surface, contraintes interfaciales... Il est également important de connaître la nature abrupte ou graduelle de la transition entre deux couches de composition chimique différente :

(*) Aide Individuelle du C.N.R.S., nº 034229. une transition abrupte se réalise sur une monocouche, et on parle alors d'interface " physique " alors qu'une transition graduelle se réalise sur plusieurs monocouches en faisant intervenir de nouvelles liaisons chimiques et l'on parle d'interface " chimique".

L'interaction mutuelle mécanique, électrique ou chimique entre les différentes couches de ces empilements rend indispensable la maîtrise de techniques de mesures non destructives et même non perturbatrices capables d'extraire des renseignements sur des couches intermédiaires sans modifier l'influence de leur environnement. De telles contraintes ont donné un regain d'intérêt aux méthodes de mesures optiques.

Du point de vue optique, une substance donnée est caractérisée par sa fonction diélectrique $\varepsilon(E)$, image de l'ensemble des transitions électroniques créées par un photon d'énergie $E$. La fonction diélectrique possède des points singuliers qui correspondent à des extrema de la structure de bandes électroniques [1]. La connaissance de la répartition en fonction de l'énergie $E$ de ces points singuliers est un moyen d'identification chimique du matériau. D'autre part, la variation de la fonction diélectrique en fonction de 
$E$, fournit des renseignements précieux sur la structure électronique des matériaux et permet de prévoir et de comprendre leurs autres propriétés dans lesquelles interviennent les mêmes électrons.

Nous présentons ici deux méthodes optiques s'appliquant parfaitement à l'étude des multicouches et donnant des renseignements complémentaires : il s'agit de l'électroréflexion et de l'ellipsométrie spectroscopique.

Après une description rapide des techniques expérimentales, nous donnons des exemples d'application à l'étude d'hétérostructures InGaAsP/InP et (GaAlAs, $\mathrm{GaAs}$ )/GaAs ayant des couches ultra-minces présentant des propriétés électroniques bidimensionnelles.

\section{Electroréflexion.}

Un spectre d'électroréflexion, donne la mesure du rapport $\frac{\Delta R}{R}=\frac{R(\mathcal{E})-R(0)}{R(0)}$ en fonction de l'énergie $E$ des photons incidents.

$R(\mathcal{E})$ est le pouvoir réflecteur en incidence normale de la surface de l'échantillon et $\delta$ le champ électrique en surface.

Pour les semiconducteurs [2], la quantité $\frac{\Delta R}{R}$ est inférieure à $10^{-3}$.

Pour mesurer ce signal il est donc nécessaire de moduler le champ électrique $\delta$ appliqué à l'échantillon et de détecter la variation correspondante du pouvoir réflecteur par des techniques de détection synchrone.

Le champ électrique $\delta$ est appliqué à partir de la barrière de Schottky formée par l'échantillon et une couche évaporée de $800 \AA$ de $\mathrm{Cu}_{2} \mathrm{~S}$ [3]. Cette épaisseur de $\mathrm{Cu}_{2} \mathrm{~S}$ permet d'effectuer des mesures entre 0,5 et 3,5 eV. L'ensemble de l'appareillage utilisé est schématisé par la figure 1 .

A «faible » champ électrique [4], la forme des spectres est indépendante du champ électrique $\mathcal{E}$ et est donnée par :

$\frac{\Delta R}{R}=\mathcal{R e}\left\{A \exp (i B) \mathcal{E}^{2}\left(E_{\mathrm{g}}-E+i \Gamma\right)^{\frac{D-8}{2}}\right\}$

où $A, B$ sont des fonctions de $E$ lentement variables, $E_{\mathrm{g}}$ l'énergie de la transition à observer, $D$ la dimension du point critique et $\Gamma$ un paramètre phénoménologique décrivant la "largeur » du spectre et relié à la durée de vie $\tau$ de la paire électron-trou par $\Gamma \cdot \tau=\hbar / 2$. $\frac{\Delta R}{R}$ est proportionnel à la dérivée troisième de la fonction diélectrique ce qui permet de mesurer avec précision l'énergie des principales transitions optiques directes qui sont pour les composés III-V :

$E_{0} \quad$ transition entre le niveau $\Gamma_{8 \mathrm{v}}$ et $\Gamma_{6 \mathrm{c}}$, $E_{0}+\Delta_{0}$ transition entre le niveau $\Gamma_{7 \mathrm{v}}$ et $\Gamma_{6 \mathrm{c}}$,

$E_{1}$ transition entre les niveaux $\mathrm{L}_{4,5 \mathrm{v}}$ et $\mathrm{L}_{6 \mathrm{c}}$, $E_{1}+\Delta_{1}$ transition entre le niveau $\mathrm{L}_{6 \mathrm{v}}$ et $\mathrm{L}_{6 \mathrm{c}}$, $E_{0}^{\prime} \quad$ transition entre le niveau $\Gamma_{8 \mathrm{v}}$ et $\Gamma_{7 \mathrm{c}}$, $E_{0}^{\prime}+\Delta_{0}^{\prime}$ transition entre le niveau $\Gamma_{8 \mathrm{v}}$ et $\Gamma_{8 \mathrm{c}}$.

A champ électrique "moyen " [5], la forme des spectres dépend du champ électrique en particulier, pour des énergies supérieures à la transition $E_{\mathrm{g}}$ apparaît les oscillations de Franz-Keldysh variant comme :

$$
A^{\prime} \mathrm{e}^{-B^{\prime}} \cos \left\{C^{\prime}+\frac{2}{3}\left(\frac{E-E_{\mathrm{g}}}{\hbar \Omega}\right)^{3 / 2}\right\}
$$

où $A^{\prime}, B^{\prime}, C^{\prime}$ sont des fonctions de l'énergie $E$ lentement variables. $\hbar \Omega$ est l'énergie électro-optique qui représente le gain d'énergie de la particule classique de masse réduite $\mu_{\|}$accélérée pendant le temps $\tau$ par le champ électrique $\delta$.

Le champ électrique $\varepsilon$ est « moyen » si $\hbar \Omega<\Gamma / 3$,

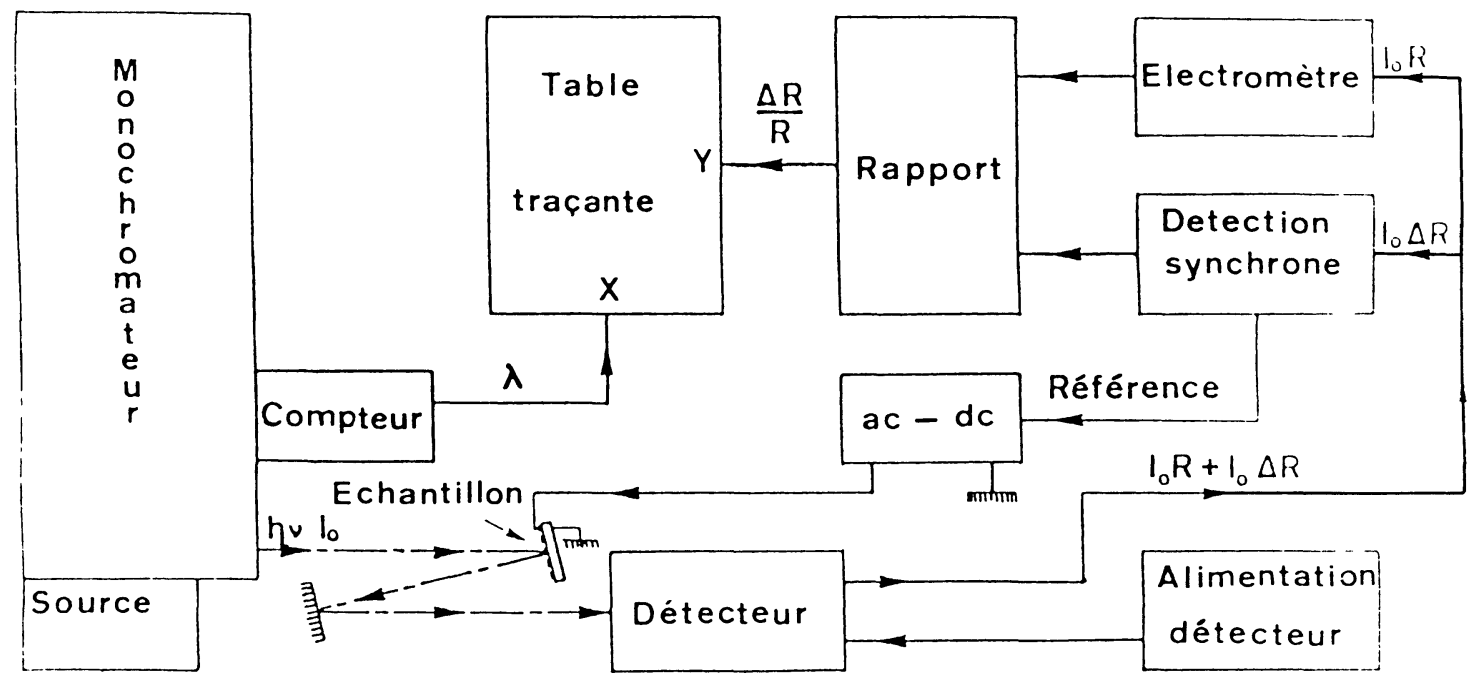

Fig. 1. - Schéma synoptique de l'expérience d'électroréflexion spectroscopique.

[Bloc diagram of the spectroscopic electroreflectance apparatus.] 
et «faible" dans le cas contraire. L'observation des oscillations de Franz-Keldysh est une mesure directe du champ électrique $\mathbb{E}$ si l'on suppose connue la masse réduite $\mu_{\|}$(parallèle à $\&$ ) donnée par :

$$
\mu_{\|}^{-1}=m_{\mathrm{e}}^{-1}+m_{\mathrm{h}}^{-1}
$$

où $m_{\mathrm{e}}$ et $m_{\mathrm{h}}$ sont respectivement les masses effectives des électrons et des trous mis en jeu par la transition optique.

\section{Ellipsométrie spectroscopique.}

L'ellipsométrie spectroscopique est une méthode optique d'analyse, basée sur la mesure de l'état de polarisation de la lumière après réflexion sur une surface plane. L'ellipsomètre spectroscopique utilisé (Fig. 2) comprend une lampe au xénon, un polariseur tournant en calcite, l'échantillon, un analyseur fixe, un monochromateur et un photomultiplicateur [6].

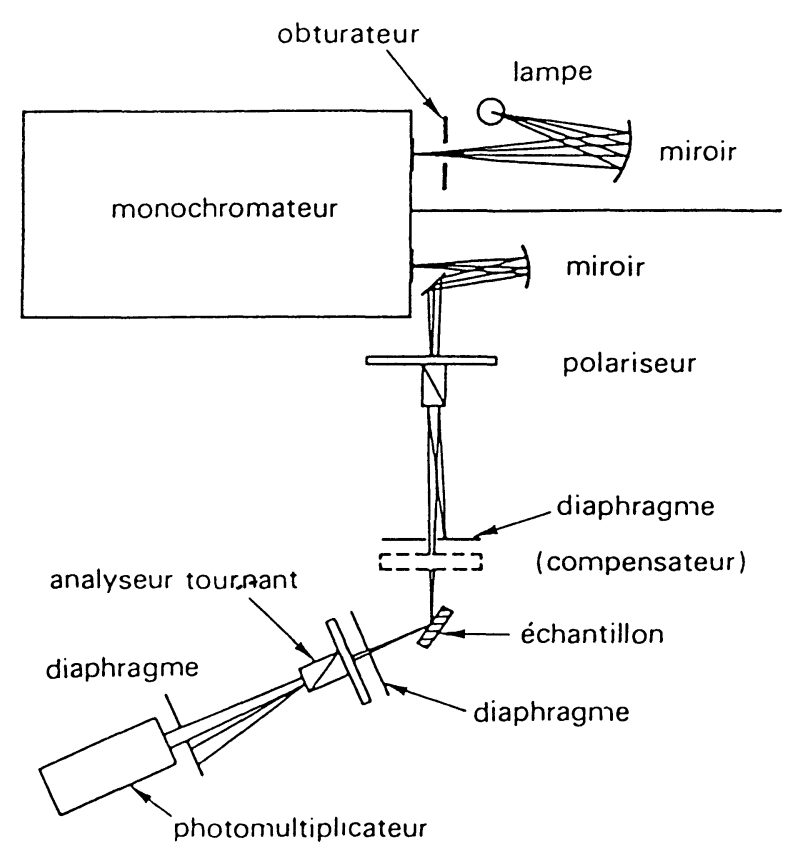

Fig. 2. - Montage expérimental de l'ellipsométrie spectroscopique.

[Experimental set-up of the spectroscopic ellipsometer.]

Il permet la mesure du rapport $\rho$ de pouvoir réflecteur complexe entre 1,6 et $5,4 \mathrm{eV}$ avec

$$
\rho=\frac{R_{\mathrm{p}}}{\boldsymbol{R}_{\mathrm{s}}}=\operatorname{tg} \psi \exp (i \Delta)
$$

où le pouvoir réflecteur $R_{\mathrm{p}}$ est obtenu par une onde lumineuse polarisée dans le plan de l'incidence et $R_{\mathrm{s}}$ par une onde lumineuse polarisée perpendiculairement au plan d'incidence. La mesure de $\rho$ en fonction de l'énergie $E$ permet d'obtenir la fonction diélectrique :

$$
\varepsilon(E)=\frac{(1-\rho)^{2}}{(1+\rho)^{2}} \sin ^{2} \theta \cdot \operatorname{tg}^{2} \theta+\sin ^{2} \theta
$$

où $\theta$ est l'angle d'incidence de la lumière sur l'échantillon.

L'ellipsométrie spectroscopique est la méthode la plus précise pour déterminer la fonction diélectrique. Comme le montre l'équation 3 , l'ellipsométrie spectroscopique compte à la fois une mesure d'amplitude $(\operatorname{tg} \psi)$ et une mesure de phase $(\cos \Delta)$, ce qui augmente notablement les informations fournies par cette technique [7].

\section{Echantillons.}

L'échantillon no 1 est un quaternaire de $\operatorname{In}_{1-x} \mathrm{Ga}_{x} \mathrm{As}_{y}$ $\mathrm{P}_{1-y}$ fabriqué par épitaxie en phase liquide sur un substrat d'InP. C'est un échantillon non intentionnellement dopé ayant une épaisseur de $1,5 \mu \mathrm{m}$. La composition déterminée par rayons $X$ vaut $x=$ $0,23 \pm 0,01, y=0,51 \pm 0,02$ avec une bonne homogénéité latérale et en profondeur. En prenant comme référence la maille $a$ de l'InP, le désaccord de maille mesuré par double diffraction $\mathrm{X}$ est faible $(\Delta a / a=$ $0,9 \times 10^{-3}$ ).

Les échantillons nos 2 à 5 à base de GaAs et de GaAlAs, ont été fabriqués par la technique d'épitaxie en phase vapeur aux organométalliques [8]. Le réacteur fonctionne à pression atmosphérique et à une température de croissance de $650{ }^{\circ} \mathrm{C}$.

La croissance s'effectue à une vitesse de $5 \AA$ par seconde à l'aide de deux flux gazeux : l'un pour la croissance du GaAs pur, l'autre pour la croissance du GaAlAs avec une composition de $54 \%$ d'aluminium. Le montage a été réalisé pour que, au-dessus du substrat, la composition des flux gazeux puisse changer de façon contrôlée en $0,1 \mathrm{~s}$.

Les échantillons $\mathrm{n}^{\circ} 2$ et $\mathrm{n}^{\circ} 3$ ont été fabriqués pour présenter une interface à $100 \AA$ de la surface.

Pour l'échantillon no 4 , une interface non abrupte a été simulée par croissance successive de dix couches de $1,5 \AA$ de $\mathrm{GaAs}+1,5 \AA$ de $\mathrm{Ga}_{0,46} \mathrm{Al}_{0,54} \mathrm{As}$. Ce procédé est équivalent à un mélange chimique puisque l'épaisseur des couches est inférieure à la monocouche. Il permet d'obtenir $30 \AA$ de $\mathrm{Ga}_{0,73} \mathrm{Al}_{0,27}$ As.

L'échantillon $n^{\circ} 5$ est un double puits quantique.

\section{Surface réelle d'un alliage GaInAsP/InP.}

L'échantillon étudié est une couche épitaxiée de GaInAsP ayant une épaisseur de $1,5 \mu \mathrm{m}$. Une mesure d'ellipsométrie spectroscopique effectuée entre 2 et $4 \mathrm{eV}$ sur l'échantillon dès sa sortie de l'enceinte de croissance permet d'obtenir la courbe $\mathrm{d}(\cos \Delta) / \mathrm{d} E$ en fonction de $E$ (Fig. 3). Cette courbe présente entre 2,7 et 3,5 eV une structure large composée de trois pics. L'existence de trois pics est ici surprenante d'autres auteurs [9] ayant montré que dans ce domaine d'énergie, il n'y a pour cette composition que deux transitions possibles $E_{1}=2,77 \mathrm{eV}, \quad E_{1}+\Delta_{1}=$ $2,99 \mathrm{eV}$.

Après décapage chimique de la surface de l'échantillon pendant $10 \mathrm{~s}$ et à $20^{\circ} \mathrm{C}$ par un mélange connu 
pour attaquer sélectivement l'InP $\left(1 \mathrm{HCl}, 1 \mathrm{H}_{3} \mathrm{PO}_{4}\right)$, la courbe $\mathrm{d}(\cos \Delta) / \mathrm{d} E$ ne présente plus que deux pics et sa forme et son amplitude sont caractéristiques de l'alliage quaternaire GaInAsP [9] (Fig. 3).

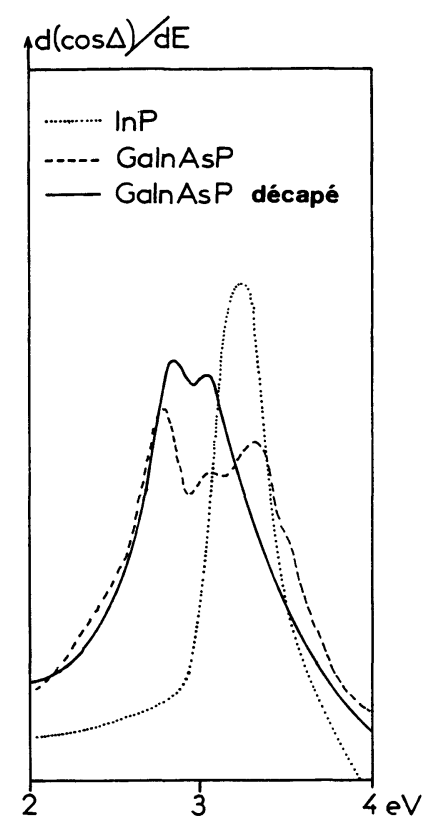

Fig. 3. - Variation de la dérivée de $\cos \Delta$ avec l'énergie pour un échantillon d'InP (en pointillés ......), un échantillon de GaInAsP non décapé (en tirets --..--.--) et le même échantillon de GaInAsP décapé (en trait plein - -).

[Cos $\Delta$ energy derivative for an InP sample (dotted line. an unetched GaInAsP sample (dashed line ---.---) and the same etched GaInAsP sample (full line - -).]

Pour mieux comprendre l'origine du troisième pic, nous avons présenté sur la figure $3, \mathrm{~d}(\cos \Delta) / \mathrm{d} E$ pour un échantillon d'InP. Cette courbe présente un maximum au voisinage de $3,2 \mathrm{eV}$ pouvant être modélisé à partir des transitions $E_{1}(3,17 \mathrm{eV})$ et $E_{1}+\Delta_{1}(3,3 \mathrm{eV})$. Compte tenu de la faible valeur de l'éclatement spinorbite $\Delta_{1}$ dans InP et des valeurs importantes des paramètres d'élargissement pour ces transitions [10], il est normal d'observer une seule structure dans la courbe de $\mathrm{d}(\cos \Delta) / \mathrm{d} E$.

Pour expliquer les valeurs de $\mathrm{d}(\cos \Delta) / \mathrm{d} E$ sur le quaternaire non décapé, nous avons calculé la fonction diélectrique en faisant l'hypothèse de l'existence sur le quaternaire d'une couche d'InP ultra-mince. La modélisation est effectuée à partir de 4 oscillateurs harmoniques : 2 centrés aux énergies $E_{1}$ et $E_{1}+\Delta_{1}$ de l'InP et les deux autres centrés aux énergies $E_{1}$ et $E_{1}+\Delta_{1}$ du quaternaire et en prenant l'épaisseur de l'InP comme paramètre ajustable à déterminer. La meilleure approximation est obtenue pour une couche d'InP ayant une épaisseur de $8,4 \AA$. L'existence de cette couche ultra-mince d'InP est normale compte tenu des conditions de croissance au stade final de la technologie de fabrication.

Sur la figure 4, nous avons représenté le spectre d'électroréflexion de l'échantillon décapé au voisinage des transitions $E_{0}$ et $E_{0}+\Delta_{0}$. Les valeurs de la transition $E_{0}(0,994 \mathrm{eV})$ et $E_{0}+\Delta_{0}(1,236 \mathrm{eV})$ obtenues par la méthode des trois points [4] sont en bon accord avec les résultats d'autres auteurs [11-13], ce qui confirme la composition chimique de ce quaternaire.

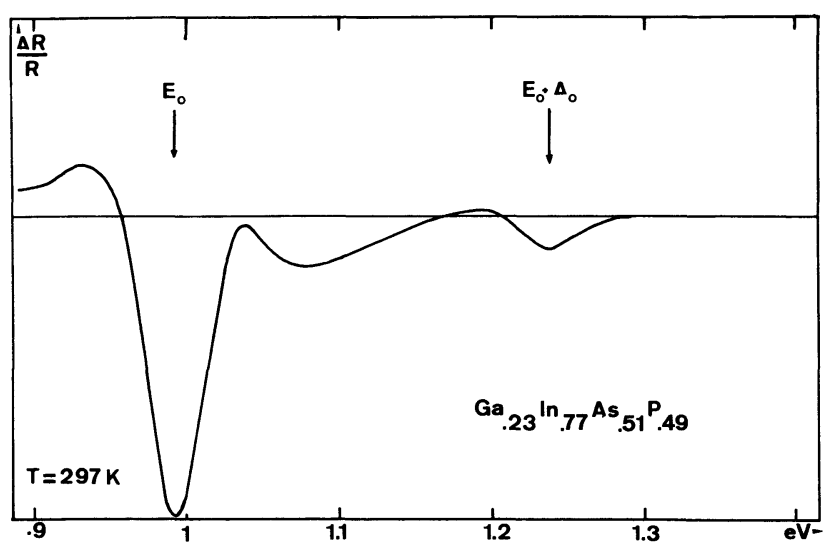

Fig. 4. - Spectre d'électroréflexion au voisinage des transitions $E_{0}$ et $E_{0}+\Delta_{0}$ de l'alliage quaternaire $\mathrm{Ga}_{0,23} \mathrm{In}_{0,77}$ $\mathrm{As}_{0,51} \mathrm{P}_{0,49}$.

[Electroreflectance spectra near the $E_{0}$ and $E_{0}+\Delta_{0}$ transitions of the $\mathrm{Ga}_{0.23} \operatorname{In}_{0.77} \mathrm{As}_{0.51} \mathrm{P}_{0.49}$ quaternary alloys.]

\section{Etude de multicouches et d'interfaces à base de GaAs et GaAlAs.}

Les échantillons décrits dans le tableau I ont été fabriqués spécialement pour présenter une interface à une profondeur de $100 \AA$ de la surface. Cette épaisseur a été choisie pour qu'au voisinage de $3 \mathrm{eV}$ seule cette interface intervienne dans les spectres d'ellipsométrie spectroscopique. Les autres interfaces, trop profondes ne jouant aucun rôle.

6. 1 ANAlySE DE L'ÉCHANTILlon No 3. - Le spectre d'électroréflexion de l'échantillon no 3 présente, entre 1,3 et $3,5 \mathrm{eV}, 6$ structures principales provenant des couches de GaAs et de GaAlAs (Fig. 5).

A basse énergie on observe entre 1,35 et $1,45 \mathrm{eV}$ une structure très aiguë provenant de la transition $E_{0}$ du GaAs. La méthode des trois points [4] permet d'obtenir l'énergie de la transition $E_{0}$ à $300 \mathrm{~K}$ : $1,420 \pm 0,003 \mathrm{eV}$. L'incertitude évaluée comme étant le dixième du paramètre d'élargissement $\Gamma$. Cette valeur de $E_{0}$ à $300 \mathrm{~K}$ est en excellent accord avec les valeurs données par Sell et al. [14] $(1,424 \mathrm{eV})$ et par Thurmond [15] $(1,422 \mathrm{eV})$.

La structure observée aux environs de $1,8 \mathrm{eV}$ a pour origine la transition $E_{0}+\Delta_{0}$ où $\Delta_{0}$ est l'énergie spin-orbite séparant les niveaux $\Gamma_{8 \mathrm{v}}, \Gamma_{7 \mathrm{v}}$. La méthode des trois points donne $E_{0}+\Delta_{0}=1,780 \pm 0,003 \mathrm{eV}$ soit $\Delta_{0}=360 \mathrm{meV}$ valeur en excellent accord avec celle mesurée par Aspnes à $4 \mathrm{~K}\left(\Delta_{0}=341 \mathrm{meV}\right)$. 
Tableạu I. - Epaisseurs et compositions désirées pour chaque échantillon à base de GaAs, GaAlAs.

\begin{tabular}{|c|c|c|c|c|c|c|c|}
\hline \multicolumn{2}{|c|}{ Echantillon $n^{\circ} 2$} & \multicolumn{2}{|c|}{ Echantillon no 3} & \multicolumn{2}{|c|}{ Echantillon $\mathbf{n}^{\circ} 4$} & \multicolumn{2}{|c|}{ Echantillon $n^{\circ} 5$} \\
\hline Nature & Epaisseur & Nature & Epaisseur & Nature & Epaisseur & Nature & Epaisseur \\
\hline GaAs & $100 \AA$ & $\begin{array}{l}\text { GaAlAs } \\
(\mathrm{Al}=0,54)\end{array}$ & $100 \AA$ & $\begin{array}{l}\text { GaAlAs } \\
(\mathrm{Al}=0,54)\end{array}$ & $60 \AA$ & $\begin{array}{l}\text { GaAlAs } \\
(\mathrm{Al}=0,54)\end{array}$ & $1000 \AA$ \\
\hline $\begin{array}{l}\text { GaAlAs } \\
(\mathrm{Al}=0,54)\end{array}$ & $10000 \AA$ & GaAs & $1000 \AA$ & $\begin{array}{l}\text { GaAlAs } \\
(\mathrm{Al}=0,27)\end{array}$ & $30 \AA$ & GaAs & $50 \AA$ \\
\hline \multicolumn{2}{|c|}{$\frac{\text { Substrat }}{\text { GaAs }}$} & $\begin{array}{l}\text { GaAlAs } \\
(\mathrm{Al}=0,54)\end{array}$ & $1000 \AA$ & GaAs & $10000 \AA$ & $\begin{array}{l}\text { GaAlAs } \\
(\mathrm{Al}=0,54)\end{array}$ & $120 \AA$ \\
\hline & & \multicolumn{2}{|c|}{$\frac{\text { Substrat }}{\mathrm{GaAs}}$} & $\begin{array}{l}\text { GaAlAs } \\
(\mathrm{Al}=0,54)\end{array}$ & $\begin{array}{l}\text { Couche } \\
\text { Tampon }\end{array}$ & GaAs & $50 \AA$ \\
\hline & & & & \multicolumn{2}{|c|}{$\frac{\text { Substrat }}{\text { GaAs }}$} & $\begin{array}{l}\text { GaAlAs } \\
(\mathrm{Al}=0,54)\end{array}$ & $\begin{array}{l}\text { Couche } \\
\text { Tampon }\end{array}$ \\
\hline & & & & & & \multicolumn{2}{|c|}{$\frac{\text { Substrat }}{\text { GaAs }}$} \\
\hline
\end{tabular}

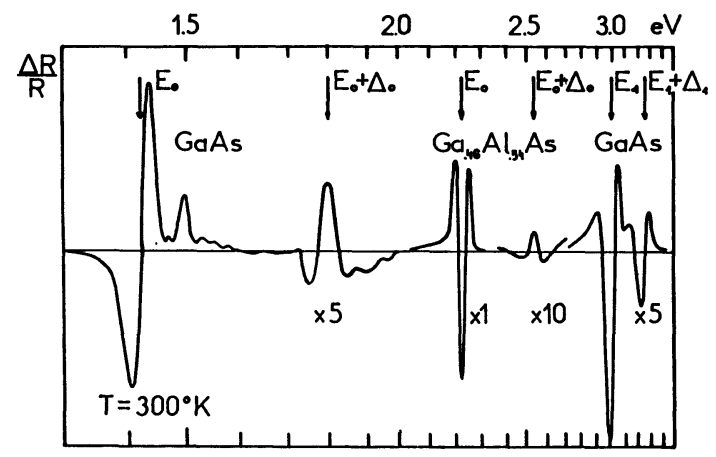

Fig. 5. - Spectre d'électroréflexion de l'hétérostructure GaAlAs, GaAs, GaAlAs/GaAs $(\mathrm{Al}=0,54)$ entre 1,3 et $3,5 \mathrm{eV}$.

[Electroreflectance spectra of the GaAlAs, GaAs, GaAlAs/ $\mathrm{GaAs}(\mathrm{Al}=0.54)$ heterostructure between 1.3 and $3.5 \mathrm{eV}$.]

Entre 2,8 et 3,5 eV, on observe le doublet $E_{1}$ et $E_{1}+\Delta_{1}$ du GaAs. La méthode des trois points donne $E_{1}=2,977 \mathrm{eV}$ et $E_{1}+\Delta_{1}=3,207 \mathrm{eV}$, la valeur de $\Delta_{1}=229 \mathrm{meV}$ donne un rapport $\Delta_{0} / \Delta_{1}=1,57 \mathrm{ce}$ qui est un excellent accord avec la valeur théorique [17] $(1,50)$.

La structure à $2,210 \mathrm{eV}$ provient de la couche superficielle et de la couche intermédiaire de GaAlAs. En effet, à $2,2 \mathrm{eV}$, les photons atteignent la couche intermédiaire puisque le coefficient d'absorption $\alpha$ du GaAs vaut $5 \times 10^{4} \mathrm{~cm}^{-1}$ et l'épaisseur $d$ du GaAs à traverser vaut $1000 \AA(\alpha d=0,5)$.

La transition $E_{0}+\Delta_{0}$ du GaAlAs est observée à $2,535 \mathrm{eV}\left(\Delta_{0}=325 \mathrm{meV}\right)$

Il ne nous a pas été possible d'observer les structures associées à $E_{1}$ et $E_{1}+\Delta_{1}$ du GaAlAs. Ceci provient du fait qu'au-dessus de $3,5 \mathrm{eV}$, la couche de $\mathrm{Cu}_{2} \mathrm{~S}$ évaporée pour créer la barrière de Schottky nécessaire aux expériences d'électroréflexion n'est plus transparente aux photons. Par contre nous verrons par la suite que ces transitions sont observables par ellipsométrie spectroscopique.

Plusieurs autres points importants ressortent de ce spectre :

- la transition $E_{0}$ du GaAlAs correspond à une composition de $54 \%$ d'aluminium, l'absence de doublet au niveau de $E_{0}$ du GaAlAs indique que les deux couches de GaAlAs ont la même réponse optique donc même composition et mêmes contraintes mécaniques ;

- l'étude en fonction du champ électrique montre que la structure " anormale " à $1,49 \mathrm{eV}$ n'est pas une oscillation de Franz-Keldysh liée à la transition $E_{0}$ du GaAs. Cette structure peut provenir d'états localisés dans le GaAs au voisinage d'une des trois hétérojonctions GaAs-GaAlAs puisque, à cette énergie, les photons peuvent pénétrer jusqu'au substrat. Cet état localisé peut être créé par un puits de potentiel triangulaire provoqué par la polarisation de l'échantillon.

Les spectres d'ellipsométrie spectroscopique (Fig. 6) de l'échantillon $\mathrm{n}^{\circ} 3$ présentent des oscillations entre 1,6 et $2,5 \mathrm{eV}$ créées par les couches de $1000 \AA$ de GaAs et GaAlAs. Dans la région 3-4 eV, on observe clairement les structures provenant des transitions $E_{1}$ et $E_{1}+\Delta_{1}$ du GaAs et du GaAlAs. Compte tenu des coefficients d'absorption, ces structures ne peuvent provenir que des couches supérieures de GaAlAs et de 
GaAs. A partir des spectres et en utilisant une méthode d'analyse multicouche, on montre que la transition GaAlAs-GaAs est abrupte, qu'elle s'effectue sur une monocouche et permet de déterminer avec précision l'épaisseur de la couche supérieure de GaAlAs (voir encart de la figure 6). Les épaisseurs des couches inférieures sont déduites des structures entre 2 et $3 \mathrm{eV}$. Le décalage entre le spectre expérimental de $\cos \Delta$ et le spectre calculé est créé par une couche d'oxyde qui croît naturellement à la surface de l'échantillon.

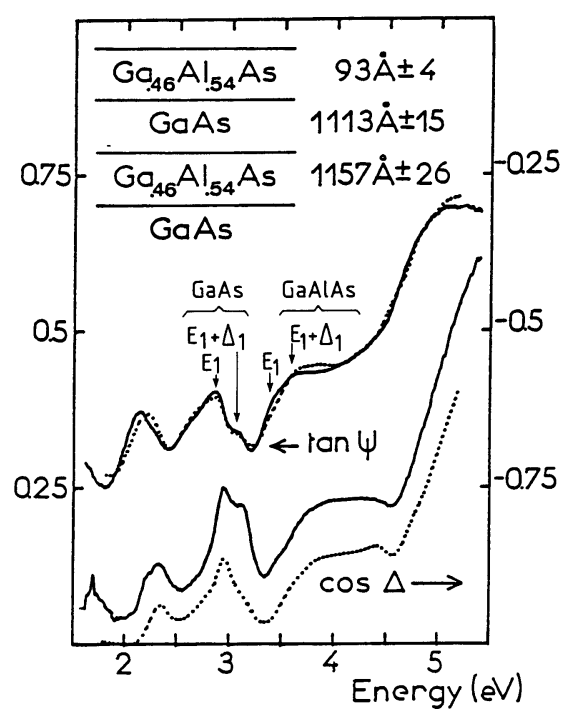

Fig. 6. - Spectres d'ellipsométrie spectroscopique (tg $\psi$ et $\cos \Delta$ ) de l'hétérostructure GaAlAs, GaAs, GaAlAs/GaAs $(\mathrm{Al}=0,54)$ sous un angle d'incidence de $66^{\circ}$. En traits pleins, les courbes expérimentales, en pointillés, les courbes calculées en prenant le modèle multicouche donné en encart.

[Spectroscopic ellipsometry measurements at $66^{\circ}$ angle of incidence of the GaAlAs, GaAs, GaAlAs/GaAs ( $\mathrm{Al}=0.54)$ heterostructure. Experimental curves (full line) and calculated curves for the multilayer model given in the inset (dotted line).]

6.2 ANALYSE DE L'ÉCHANTILlON No 2. - Le spectre d'électroréflexion de l'échantillon $n^{\circ} 2$ (Fig. 7) a une allure très différente du spectre d'électroréflexion de l'échantillon no 3.

A basse énergie, la structure $E_{0}$ du GaAs présente de larges oscillations de Franz-Keldysh provenant d'une zone où il existe un champ électrique de l'ordre de $250 \mathrm{kV} / \mathrm{cm}$. Ces oscillations ne peuvent avoir pour origine l'hétérojonction GaAlAs-substrat puisque pour l'échantillon no 3 préparé de façon analogue, nous n'avons observé sur le spectre de la figure 5 qu'une faible valeur du champ électrique.

Le spectre «fort champ électrique » de la figure 7 a donc pour origine la couche supérieure de GaAs. D'autre part, on observe au voisinage de $1,4 \mathrm{eV}$ une structure que l'on peut attribuer à des niveaux d'impureté. Ces deux observations prouvent que la couche superficielle de GaAs est de mauvaise qualité élec- trique ou que la première interface GaAs-GaAlAs n'est pas abrupte.

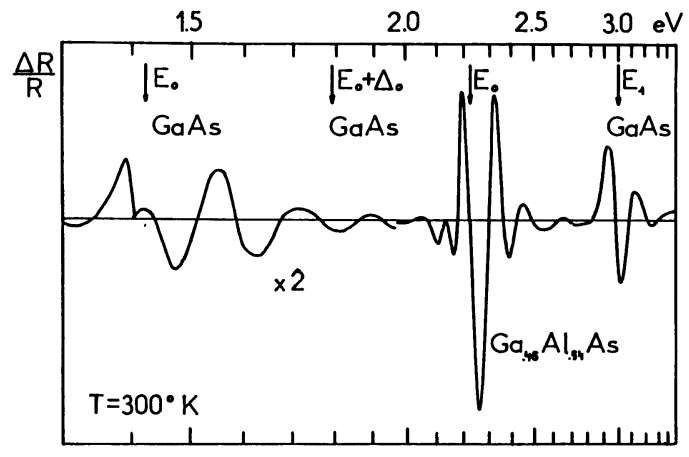

Fig. 7. - Spectre d'électroréflexion de l'hétérostructure GaAs, GaAlAs/GaAs $(\mathrm{Al}=0,54)$ entre 1,3 et $3,5 \mathrm{eV}$.

[Electroreflectance spectra of the GaAs, GaAlAs/GaAs $(\mathrm{Al}=0.54)$ heterostructure between 1.3 and $3.5 \mathrm{eV}$.]

La transition $E_{0}$ du GaAlAs $(\mathrm{Al}=0,54)$ est en fait un doublet. Une étude détaillée de ce doublet montre l'existence de contraintes dans la couche épaisse de GaAlAs. Ce phénomène est bien connu dans le cas des couches épaisses de GaAlAs épitaxiées sur GaAs et a déjà été observé en électroréflexion [20].

Les spectres d'ellipsométrie spectroscopique (Fig. 8) de l'échantillon no 2 présentent de nombreuses oscillations au voisinage de $2 \mathrm{eV}$. Ces oscillations dues à des interférences permettent de déterminer l'épaisseur de la couche tampon de GaAlAs $(10500 \pm 100 \AA ̊)$.

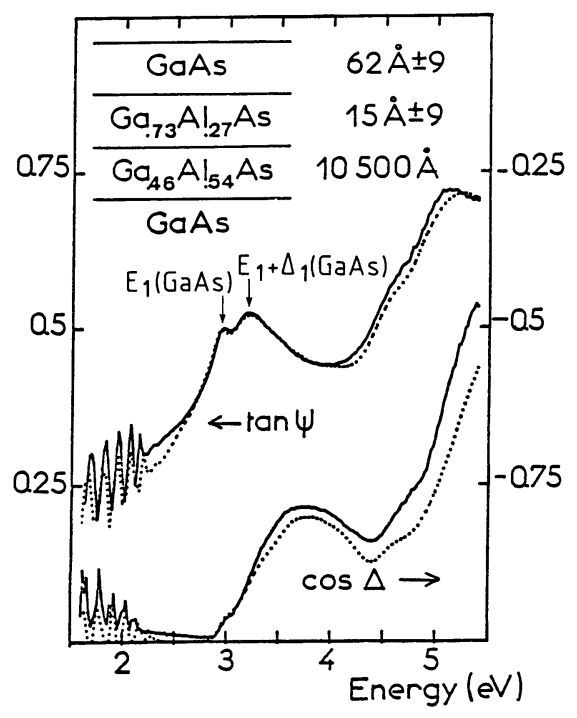

Fig. 8. - Spectres d'ellipsométrie spectroscopique (tg $\psi$ et $\cos \Delta)$ de l'hétérostructure $\mathrm{GaAs}, \mathrm{GaAlAs} / \mathrm{GaAs}(\mathrm{Al}=0,54)$ sous un angle d'incidence de $66^{\circ}$. En traits pleins, les courbes expérimentales, en pointillés, les courbes calculées en prenant le modèle multicouche donné en encart.

[Spectroscopic ellipsometry measurements at $66^{\circ}$ angle of incidence of the GaAs, GaAlAs/GaAs (Al = 0.54) heterostructure. Experimental curves (full lines) and calculed curves for the multilayer model given in the inset (dotted line).] 
Au voisinage de $3 \mathrm{eV}, \operatorname{tg} \psi$ présente un doublet qui a pour origine la couche supérieure ultra-mince de GaAs. Une analyse multicouche prouve qu'il existe une région d'interface entre la couche superficielle de GaAs et la couche tampon de GaAlAs. La meilleure estimation est obtenue pour une couche de GaAs de $62 \pm 9 \AA$ avec une interface chimique de GaAlAs contenant $27 \%$ d'aluminium de $15 \pm 9 \AA$. L'existence de cette interface chimique est à l'origine du fort champ électrique observé par électroréflexion dans la couche superficielle de GaAs. L'existence d'une couche d'oxyde à la surface du GaAs provoque le décalage observé entre la courbe expérimentale de $\cos \Delta$ et la couche théorique de $\cos \Delta$.

6.3 ANALYSE DE L'ÉCHANTILlON No 4. - L'échantillon no 4 a été fabriqué pour présenter une interface chimique de $30 \AA$ de GaAlAs $(\mathrm{Al}=0,27)$ entre une couche ultra-mince de $60 \AA$ de $\operatorname{GaAlAs}(\mathrm{Al}=0,54)$ et la couche tampon de GaAs.

Sur le spectre d'électroréflexion de cet échantillon (Fig. 9) on observe : les transitions $E_{0}$ et $E_{0}+\Delta_{0}$ du $\mathrm{GaAs}$, et deux structures, l'une provenant de la couche intermédiaire de $30 \AA$ (à $1,67 \mathrm{eV})$, l'autre provenant de la couche supérieure de $60 \AA$ (à $1,96 \mathrm{eV}$ ). Cette dernière structure présente nettement toutes les caractéristiques d'un spectre d'électroréflexion à «faible champ ".

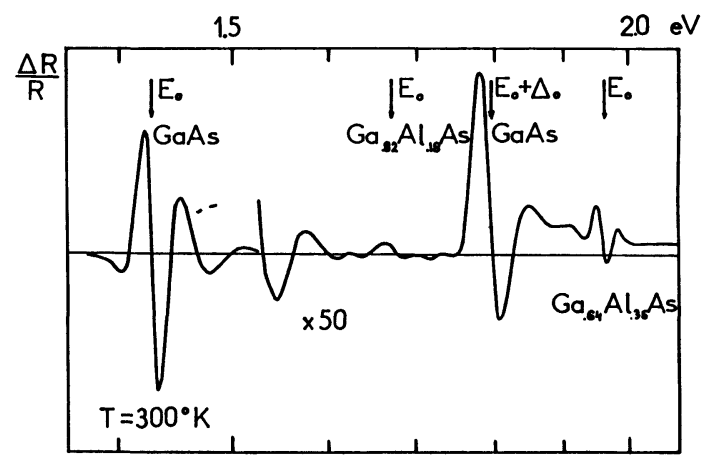

Fig. 9. - Spectre d'électroréflexion de couches ultra minces de $\mathrm{GaAl}_{(1)} \mathrm{As} \mathrm{GaAl}_{(2)} \mathrm{As} / \mathrm{GaAs},\left(\mathrm{Al}_{(1)}=0,54, \mathrm{Al}_{(2)}=0,27\right)$ entre 1,35 et $2,1 \mathrm{eV}$.

[Electroreflectance spectra of the $\mathrm{GaAl}_{(1)} \mathrm{As}, \mathrm{GaAl}_{(2)} \mathrm{As} /$ $\mathrm{GaAs}$ ultra thin layers between 1.35 and $2.1 \mathrm{eV},\left(\mathrm{Al}_{(1)}=0.54\right.$, $\left.\mathrm{Al}_{(2)}=0.27\right)$.]

En prenant comme hypothèse que cette couche ultramince de GaAlAs a la même structure de bande que le matériau tridimensionnel, on obtient une composition chimique en aluminium de $36 \%$ ce qui s'écarte notablement de la composition prévue de $54 \%$. Avec les mêmes hypothèses bien que le pic à $1,97 \mathrm{eV}$ soit mélangé aux oscillations de FranzKeldyh provenant de la transition $E_{0}$ du substrat, on en déduit que la couche intermédiaire contient $18 \%$ d'aluminium. On remarque que le facteur 2 prévu' par le procédé de fabrication entre les compositions chimiques des deux couches est conservé.

Ce spectre prouve qu'une couche ultra-mince de $30 \AA$ peut être détectée et qu'une couche ultra-mince de $60 \AA$ de GaAlAs donne en électroréflexion et pour la transition $E_{0}$ une structure étroite parfaitement définie.

Pour analyser les spectres d'ellipsométrie spectroscopique de la figure 10, deux modèles ont été utilisés. L'un suppose que la couche intermédiaire est une interface physique de $50 \%$ de GaAs et de $50 \%$ de GaAlAs $(\mathrm{Al}=0,54)$, l'autre une interface chimique ayant une composition d'aluminium de $27 \%$. Le deuxième modèle permet de mieux approcher les courbes expérimentales et d'apprécier avec une meilleure précision les épaisseurs des différentes couches. Ces épaisseurs sont données par l'encart de la figure 10

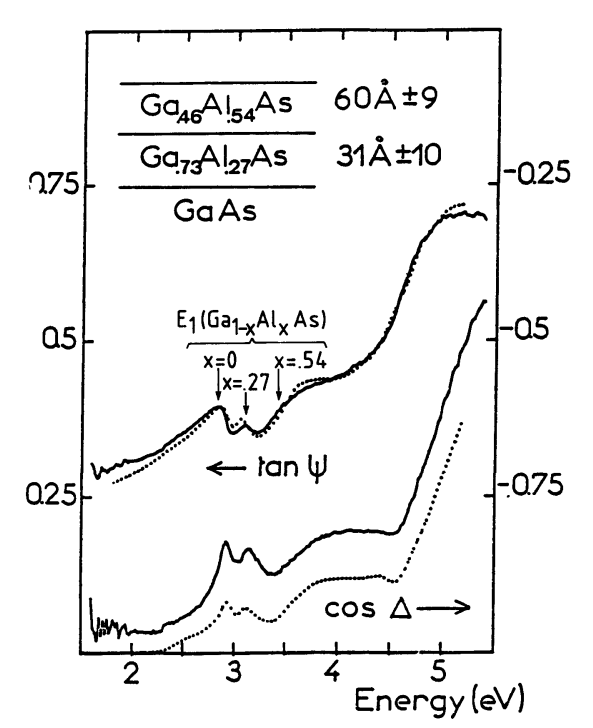

Fig. 10. - Spectres d'ellipsométrie spectroscopique $(\operatorname{tg} \psi$ et $\cos \Delta$ ) de couches ultra minces de $\mathrm{GaAl}_{(1)} \mathrm{As}, \mathrm{GaAl}_{(2)} \mathrm{As} /$ $\mathrm{GaAs},\left(\mathrm{Al}_{(1)}=0,54, \mathrm{Al}_{(2)}=0,27\right)$, sous un angle d'incidence de $77^{\circ}$. En traits pleins, les courbes expérimentales, en pointillés, les courbes calculées en prenant le modèle multicouche donné en encart.

[Spectroscopic ellipsometry measurements at $77^{\circ}$ angle of incidence of the $\mathrm{GaAl}_{(1)} \mathrm{As}, \mathrm{GaAl}_{(2)} \mathrm{As} / \mathrm{GaAs}$ ultra-thin layers. $\left(\mathrm{Al}_{1}=0.54, \mathrm{Al}_{2}=0.27\right)$. Experimental curves (full line) and calculed curves for the multilayer model given in the inset (dotted line).]

Les compositions des couches prisent dans le modèle $(27 \%$ et $54 \%$ d'aluminium $)$ diffèrent de celles trouvées en électroréflexion. En fait, pour analyser les spectres d'ellipsométrie spectroscopique ces compositions n'ont pas été prises comme paramètre ajustable puisque de façon générale l'ellipsométrie spectroscopique est moins sensible à la composition que l'électroréflexion. Par contre, l'ellipsométrie spectroscopique permet seule d'évaluer les épaisseurs des différentes couches. 


\section{Double puits quantique à base de GaAs dans une matrice de GaAlAs.}

Les deux puits quantiques de GaAs ont une épaisseur prévue de $50 \AA$, ils sont séparés par $120 \AA \AA$ de GaAlAs $(\mathrm{Al}=0,54)$ et contenus dans une matrice de GaAlAs $(\mathrm{Al}=0,54)$. L'épaisseur de la couche intermédiaire de GaAlAs (120 $\AA$ ) permet de considérer que ces deux puits quantiques sont découplés.

Le spectre d'électroréflexion (à $35 \mathrm{~K}$ ) et de photoluminescence (à $4 \mathrm{~K}$ ) sont reportés figure 11 . Le spectre de photoluminescence a été obtenu à l'aide de la raie 514,5 $\mathrm{nm}$ d'un laser argon avec une densité d'excitation de l'ordre de $0,4 \mathrm{~W} \mathrm{~cm} \mathrm{~cm}^{-2}$. L'énergie du pic de photoluminescence $(1,66 \mathrm{eV})$ correspond à la transition trous lourds $(n=1) \rightarrow$ électrons $(n=1)$ du puits quantique. Le spectre d'électroréflexion est beaucoup plus riche d'information, il présente entre 1,4 et 2,4 $\mathrm{eV} 7$ structures principales.

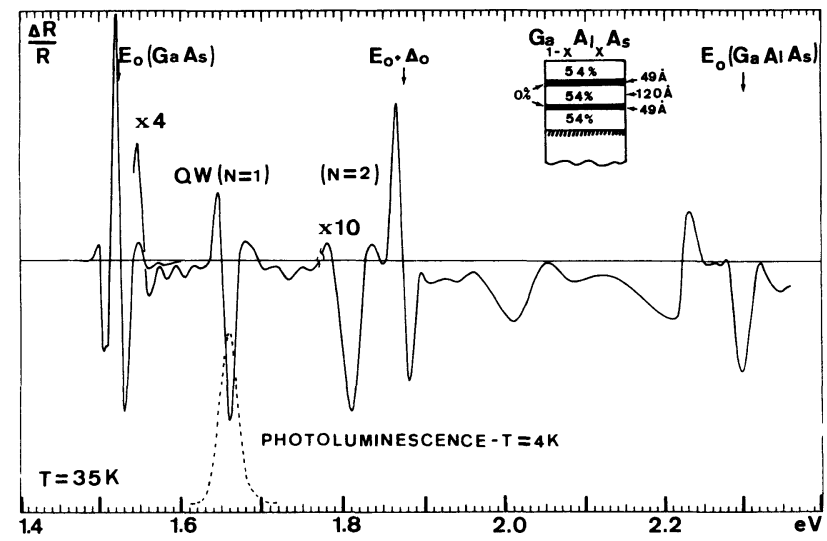

Fig. 11. - Spectre d'électroréflexion et de photoluminescence entre 1,4 et $2,4 \mathrm{eV}$ du double puits quantique décrit par l'encart.

[Electroreflectance and photoluminescence spectra of the double quantum well given in the inset between 1.4 and $2.4 \mathrm{eV}$.]

Trois structures $(1,5 \mathrm{eV}, 1,85 \mathrm{eV}$ et $2,2 \mathrm{eV})$ proviennent du substrat $\left(E_{0}, E_{0}+\Delta_{0}\right)$ et de la matrice de $\operatorname{GaAlAs}\left(E_{0}\right)$.
Les autres structures $(1,65 \mathrm{eV}, 1,8 \mathrm{eV}, 2 \mathrm{eV}, 2,07 \mathrm{eV})$ ont pour origine les niveaux électroniques des 2 puits quantiques : 2 niveaux pour les électrons, 4 niveaux pour les trous de la bande de valence ( 2 niveaux pour les trous lourds et 2 niveaux pour les trous légers). De plus, la structure observée à $2 \mathrm{eV}$ correspond à la transition entre les niveaux des trous de la bande $\Gamma_{7}$ et le niveau $n=1$ des électrons de la bande de conduction.

Les oscillations de Franz-Keldysh observables pour les énergies supérieures à $E_{0}$ du GaAs montrent qu'il existe à l'interface GaAs (substrat)-GaAlAs un champ électrique de l'ordre de $30 \mathrm{kV} \mathrm{cm}{ }^{-1}$.

La largeur et la position des spectres d'électroréflexion provenant des puits quantiques confirment [21] pour ce type d'échantillon que la brusque transition de composition s'effectue sur $5 \AA$ environ.

\section{Conclusion.}

Nous avons montré que l'électroréflexion et l'ellipsométrie spectroscopique sont des outils puissants pour obtenir des informations détaillées sur les structures multi-couches.

L'électroréflexion permet de mesurer l'énergie des principales transitions optiques, de mesurer la composition chimique d'une couche ultra-mince de $30 \AA$ située à $60 \AA$ de la surface, d'évaluer des champs électriques d'interface, de montrer l'existence de contraintes mécaniques entre couches et d'observer une série de niveaux électroniques dans un double puits quantique.

L'ellipsométrie spectroscopique permet de mesurer l'énergie des transitions optiques situées au-dessus du gap, d'obtenir les constantes optiques, d'évaluer la composition chimique, de mesurer les épaisseurs des différentes couches avec une précision pouvant atteindre la monocouche et d'apprécier la nature physique ou chimique des interfaces quand celles-ci ne sont pas abruptes.

Enfin, il faut souligner que ces deux méthodes spectroscopiques sont non destructives et complémentaires puisque l'électroréflexion donne ses principaux résultats entre 0,5 et $3 \mathrm{eV}$ et l'ellipsométrie entre 2 et $5,5 \mathrm{eV}$.

\section{Bibliographie}

[1] Harbeke, G., Optical Properties of Solids, F. Abelès ed. (North Holland Co.) 1972.

[2] Cardona, M., Modulation Spectroscopy, Solid State Physics Suppl. 11 (Academic Press, New York) 1969.

[3] Alibert, C., Jacquemin, J. L., Bordure, G., J. Phys. E 5 (1972) 249.

[4] Aspnes, D. E. et Rowe, J. E., Phys. Rev. Lett. 27 (1971) 188.

[5] Aspnes, D. E., Phys. Rev. Lett. 31 (1973) 230.

[6] Aspnes, D. E., Studna, A. A., Rev. Sci. Instrum. 49 (1978) 291 ;

Aspnes, D. E., Studna, A. A., Appl. Opt. 14 (1975) 220.
[7] Abeles, F., Acta Electronica 24-2 (1981/1982) 133.

[8] Frijlink, P. M. and Maluenda, J., Jpn. J. Appl. Phys. 21 (1982) L574.

[9] Kelso, S. M., Aspnes, D. E., Pollack, M. A. and NAHORY, R. E., Phys. Rev. B 26 (1982) 6669.

[10] Alibert, C., Bordure, G., Laugier, A. and CheVAllier, J. Phys. Rev. B 6 (1972) 1301.

[11] Perea, E. H., Mendez, E. E., Fonstad, C. G., Appl. Phys. Lett. 36 (1980) 978.

[12] Yamazoe, Y., Nishino, T., Hamakawa, Y., I.E.E.E. J. Quantum Electron QE 17 (1981) 139.

[13] Pearsall, T. P., GaInAsP Semiconductors, ed. par T. P. Pearsall (John Wiley \& Sons Ltd) 1982, p. 299. 
[14] Sell, D. D., Stokowski, S. E., Dingle, R. and Dilorenzo, J. V., Phys. Rev. B 7 (1973) 4568.

[15] Thurmond, C. D., J. Electrochem. Soc. 122 (1975) 1133.

[16] Aspnes, D. E. and Studna, A. A., Phys. Rev. B 7 (1973) 4605.

[17] Cardona, M., Proc. of the Int. Conf. on the Physics of Semiconductors Paris, ed. by M. Hulin (Dunod, Paris) 1964 , p. 181

[18] Casey, H. C., Sell, D. D. and Wecht, K. W., J. Appl. Phys. 46 (1975) 250.
[19] Erman, M., Theeten, J. B., Vodjdani, N. and Demay, Y., Proc. of the Int. Conf. on Metastable and Modulated Semiconductor Structures, Pasadena, Californie (Dec. 1982).

[20] Pollak, F. H. and Woodall, J. M., J. Vac. Sci. Technol. 17 (1980) 1108.

[21] Frijlink, P. M. and Maluenda, J., Jpn J. Appl. Phys. 21 (1982) L574. 